Revue d'histoire de l'Amérique française

ZRS REVUE D.HISTOIRE DE L'AMÉRIQUE FRANÇAISE

\title{
Le ministère de Félix-Gabriel Marchand (1897)
}

Bernard Chevrier

Volume 22, numéro 1, juin 1968

URI : https://id.erudit.org/iderudit/302751ar

DOI : https://doi.org/10.7202/302751ar

Aller au sommaire du numéro

Éditeur(s)

Institut d'histoire de l'Amérique française

ISSN

0035-2357 (imprimé)

1492-1383 (numérique)

Découvrir la revue

Citer cet article

Chevrier, B. (1968). Le ministère de Félix-Gabriel Marchand (1897). Revue

d'histoire de l'Amérique française, 22(1), 35-46. https://doi.org/10.7202/302751ar d'utilisation que vous pouvez consulter en ligne.

https://apropos.erudit.org/fr/usagers/politique-dutilisation/ 


\section{LE MINISTĖRE DE FÉLIX-GABRIEL MARCHAND (1897)}

\section{L'élection de mai 1897; la formation du ministère; \\ le programme $d u$ parti}

Pour bien comprendre et saisir toute l'ampleur du changement qui s'opéra sur la scène provinciale, dans les dernières années du $19^{\mathrm{e}}$ siècle, il est essentiel de porter son regard sur la scène fédérale, centre d'une profonde évolution. Avec l'arrivée au pouvoir de sir Wilfrid Laurier, le 13 juillet 1896, une vague de libéralisme économique et politique, encore partiellement voilée, se manifesta.

\section{Sur la scène fédérale}

À l'échelon politique, Laurier a su réaliser un habile tour de force qui, jusqu'à nos jours, épate encore les politicologues les plus avérés. Il fait entrer dans son ministère, à titre de ministre des travaux publics, un ancien "bleu", Israël Tarte, qu'on surnommera bientôt "le chef de l'administration". Avec l'appui de ce dernier, ainsi que celui du lieutenant-gouverneur de la province, pour l'époque, sir J.-A. Chapleau qui est également un converti de dernière heure à la cause de Laurier, les conservateurs du Québec sont réduits à l'aile négligeable des "castors bleus". Une sorte de consensus provincial en faveur du premier ministre s'établira, lui permettant de gouverner jusqu'en 1911, sans crainte d'une opposition québécoise sérieuse et concertée.

$\mathrm{Au}$ domaine financier, Laurier allait réaliser un autre exploit non moins spectaculaire que le premier, en délaissant les magnats financiers du Canadien Pacifique, pour se tourner vers les dirigeants de la compagnie du chemin de fer du Grand Tronc, en leur livrant le trafic du chemin de fer Intercolonial, 
allant de Halifax à Montréal, ainsi qu'une partie du fief océanique Londres-Québec.

Les magnats britanniques, profitant d'un climat qui s'annonçait désormais favorable et rentable, permettent l'affluence de capitaux à des taux d'intérêts ridiculement bas. Désormais, le gouvernement fédéral peut contracter un emprunt à longue échéance, au taux avantageux de $2.88 \%$. Plusieurs projets d'envergure nationale vont s'amorcer, ou se continuer, dont l'emploi de wagons réfrigérés pour transporter les viandes de l'ouest canadien vers les ports des Grands Lacs et du St-Laurent. Les plans pour creuser le canal Soulanges, qui devait remplacer celui de Beauharnois et relier le lac Saint-François au lac Saint-Louis, étaient déjà tracés. Depuis quelques années, la production du blé de l'Ouest apparaissait suffisamment progressive pour que l'on puisse parler d'améliorer le réseau des canaux pour ravitailler le port de Montréal et acheminer ces produits agricoles des plaines de l'Ouest vers l'Angleterre. Le creusage de Soulanges n'était qu'un des jalons de ce qu'allait devenir ce vaste réseau de canalisation maritime.

Laurier voyait loin. Un tel rêve d'expansion faisait également frémir plus d'un financier. Mais comment allait-il réaliser ces nombreux projets? Car durant ce temps, le Québec, sous l'administration d'Edmund Flynn, faisait la sourde oreille à tout projet d'envergure nationale. Et, sans l'appui provincial, aucune possibilité pour Ottawa d'établir une ligne de "steamers" rapides entre Londres et Québec, ni aucune chance de construire un pont à Québec, afin de prolonger l'Intercolonial.

La chance unique se présenta à l'élection provinciale du 11 mai 1897, alors que l'équipe libérale de F.-G. Marchand faisait la lutte à l'équipe sortante d'Edmund Flynn. Même si le résultat de cette élection n'affectait en rien la répartition des sièges à l'échelle fédérale, Laurier connaissait fort bien toute la portée psychologique qu'une défaite libérale provinciale aurait chez ses confrères ontariens. Il n'était pas sans se douter que ces derniers auraient vite conclu qu'il était destiné à subir le 
même sort que la lignée des chefs conservateurs qui s'étaient succédé au pouvoir depuis la mort de sir John A. Macdonald en 1891, jusqu'à son propre avènement en juillet 1896. La chance était unique; il n'allait pas la rater. Les chefs de file québécois les plus imposants, disons Tarte, Fitzgerald, Joly, Fisher et Lemieux, furent lancés dans la bataille. Ce contingent venant imposer sa présence, imposition d'ailleurs bienvenue dans le camp libéral, faisait dire au journal La Vérité:

Ce n'est plus le gouvernement du Québec qui gouverne la province, c'est le gouvernement d'Ottawa, puisque c'est lui qui fait nos élections provinciales. ${ }^{1}$

$\mathrm{Au}$ lendemain de la victoire, le nouveau premier ministre F.-G. Marchand se sentit obligé de démontrer ouvertement sa dette de gratitude envers Ottawa en disant:

nous arborerons le drapeau à côté de notre chef qui gouverne à Ottawa. Laurier peut désormais commander à toutes les provinces, sa parole sera écoutée et ses désirs exécutés. ${ }^{2}$

Pourtant, il s'agissait d'une victoire provinciale, mais les libéraux fédéraux, $\mathrm{y}$ ayant tellement trempé, se sentirent autant vainqueurs que les vainqueurs eux-mêmes. Nulle autre explication n'est donc nécessaire pour comprendre l'ingérence de Tarte et de Laurier dans la formation du cabinet Marchand, du moins en ce qui a trait à la nomination de certains ministres, à l'éloignement d'autres et à l'orientation de certaines politiques économiques, d'intérêt censément national.

\section{Dispute autour de la nomination du lieutenant-gouverneur}

Dès la formation du gouvernement, la nomination du lieutenant-gouverneur fut une profonde cause de discorde, en plus d'être, parmi tant d'autres, une première ingérence du fédéral en matière provinciale. Bien qu'elle vienne d'Ottawa, cette nomination devait refléter et respecter les vues de la province en question. Pour Israël Tarte, il n'y avait aucune discussion pos-

\footnotetext{
1 Journal La Vérité, 22 mai 1897, "Fédéral et provincial", 3, col. 3.
}

2 La Patrie, mai 1897. 
sible à ce sujet. Son vouloir était loi. Son ami, Adolphe Chapleau, devait conserver son poste. Depuis quelque temps, les deux travaillaient, de concert, avec l'appui secret de Laurier, pour tenter de rallier à la cause libérale les extrémistes du parti, les "castors rouges", de même que les ardents conservateurs, les "castors bleus". Le chat allait bientôt sortir du sac. A l'insu de nos deux amis en cause, on allait rendre publique une lettre dans laquelle Chapleau expliquait à Tarte qu'il était obligé de limoger, parmi les siens, certains personnages qui étaient devenus trop influents. En réponse, Tarte se plaignit du même mal chez certains membres de son nouveau parti d'adoption et approuvait le remède proposé par Chapleau. Devant cette soudaine révélation d'une politique de sourdine qu'on avait réussi à camoufler jusqu'à ce jour, les libéraux de vieille souche, s'inquiétant de voir deux convertis de dernière heure à la cause libérale prendre autant d'importance auprès de leur chef, s'agitèrent. La révolte grondait et, si Laurier ne décida pas de renvoyer les deux indésirables, la scission semblait proche et le dénouement n'allait pas tarder. François Langelier, à qui Laurier avait promis le poste de lieutenant-gouverneur, fort de l'appui des révoltés du parti mais las d'attendre plus longtemps sa nomination qui tardait à venir, anéantit à tout jamais ses chances d'accéder à ce haut poste en s'attaquant à Laurier, disant:

Il (Laurier) n'est pas apte à gouverner. Il nomme McPherson comme commissaire du havre, qui était secrétaire de l'organisation conservatrice qui me fit la lutte dans mon comté. ${ }^{3}$

Le candidat que les révoltés proposaient comme successeur de Chapleau détruisait ses propres chances. Laurier lui-même se chargea de mater les pressions pour déloger Tarte, en se déclarant solidaire de ce dernier:

Si Tarte quitte, je quitte aussi. Sans Tarte, je ne serais pas premier ministre aujourd'hui. ${ }^{4}$

Par respect pour leur chef, les libéraux s'apaisent. L'agitation aura quand même porté ses fruits car Chapleau est dépêché en

${ }^{3}$ L’Evénement, déc. 1897.

${ }^{4} L$ L'vénement, déc. 1897. 
mission à Washington. Jetté le remplace et, malgré son intempérance, Langelier accède au banc. Personne n'aura toutefois réussi à éloigner Israël Tarte du piédestal sur lequel le tenait Laurier.

\section{Le ministère Marchand}

Certains problèmes épineux se posent à la formation de la nouvelle équipe libérale; entre autres, les cas de J.-E. Robidoux, de F.-X. Lemieux et d'Horace Archambault.

Aucune intention ne se manifeste, de la part du premier ministre, d'éloigner la présence de Robidoux du ministère. Pour les jeunes et pour les admirateurs de Mercier, il représentait ce qu'il y avait encore de plus éclairé dans la pensée libérale de l'époque. A sa façon, il symbolisait ce que Mercier avait jadis été, quelques années auparavant, c'est-à-dire l'inspirateur d'une pensée nationale toujours latente, capable d'éclater à tout moment pour la défense du fait français. On lui remettait la tâche de continuer et d'animer cette flamme sacrée. Marchand craignait tout simplement d'élever cet ardent défenseur à un poste clé, de peur de trop identifier son régime à celui de Mercier. Peut-être aussi craignait-il de voir son influence politique, auprès d'un vaste secteur de l'électorat, demeurer dans l'ombre de son jeune député car, dès le lendemain des élections, un groupe de fervents lançaient une campagne afin de valoriser la cause de leur émule. Quatorze députés auraient signé un "round robin" exigeant la nomination de Robidoux à un poste clé sans quoi ils se seraient montrés hostiles envers le ministère. Le bouillant chef de file nationaliste entre au cabinet humilié, car il n'y accède qu'à titre de secrétaire de la province, poste peu convoité.

F.-X. Lemieux, tout comme Robidoux, jouissait également d'une très grande popularité auprès de l'électorat. Lui aussi s'était taillé une réputation enviable, non seulement dans la province mais à travers le pays, en plaidant devant les tribunaux de Régina la cause de Louis Riel, en 1885. Quelques années plus tard, Mercier, accusé d'avoir fraudé les fonds de l'état d'une valeur de $\$ 60,000$., avait recours à ses 
services pour être exonéré. A l'élection partielle de 1894, en remportant le siège de Bonaventure, Lemieux devenait le précurseur de la montée du libéralisme; car, déjà, il annonçait le grand tournant de 1896 qui allait porter Laurier au pouvoir. Voguant et profitant d'une popularité à son apogée, Lemieux remporta à l'élection provinciale de 1897 une double victoire dans Lévis et Bonaventure. Comment Marchand pouvait-il se passer de ses talents oratoires de beaucoup supérieurs à ceux de Turgeon, de Dechêne et d'Archambault ? Comment pouvait-il éloigner du ministère celui qui avait si bien servi ses maîtres d'Ottawa et de Québec ? Pourtant, il le fit. C'est qu'à un moment où le climat politique invitait à la concession et aux rapprochements politiques, Lemieux eut malheureusement la faiblesse de demeurer un vrai "rouge" pendant que son chef d'Ottawa s'avérait opportuniste et cherchait à se consolider au pouvoir, en tissant des liens avec les conservateurs ainsi qu'avec l'épiscopat qu'on avait malmenés aux dernières élections. Un intransigeant de la trempe de Lemieux, qui aurait pu facilement contrarier de telles alliances, encore et toujours très fragiles, devait être éloigné discrètement. Pour cette raison, lors d'une réunion secrète de deux premiers ministres à Montréal, on s'accorda pour écarter Lemieux en le nommant juge de la Cour Supérieure.

Derrière la nomination d'Horace Archambault au poste de procureur général, au détriment de celle de F.-X. Lemieux, on voit le rôle en coulisse de l'éminence grise, Israël Tarte. Advenant une baisse soudaine de son influence à Ottawa, ce dernier désirait s'assurer une agréable porte de sortie en venant se réfugier dans l'ombre de son ami Archambault qu'il aida à entrer au ministère et qu'il voyait devenir premier ministre dans quelques années. Par contre, il semble que le jeune Archambault démontrait, depuis plusieurs années, des qualités personnelles remarquables, ayant succédé à son père au Conseil législatif en 1888, à l'âge de 31 ans et étant devenu, presqu'aussitôt, le chef de son parti dans cette Chambre.

En faisant de son ministère une synthèse des tendances ethniques, religieuses et politiques de l'époque, F.-G. Marchand 
démontra qu'il était à la fois le chef conciliant et clairvoyant. Conciliant parce qu'il savait bien que, s'il désirait à tout prix sortir sa province du marasme économique et scolaire qui la minait depuis longtemps, il avait besoin de l'appui de tous les secteurs provinciaux. Et cet appui, il le trouva en unissant dans un même ministère les éléments les plus disparates. C'est ainsi qu'Irlandais et Anglais catholiques et protestants vinrent coudoyer les Canadiens français catholiques à la salle des délibérations; que des gens de la haute finance de Montréal et de Québec, tels MM. G. W. Stephens, J. Sheyn et S.-N. Parent, ayant intérêt à maintenir l'inégalité des biens et leur emprise privilégiaire sur les nombreuses concessions d'eau et de terres de la province, durent faire bon ménage avec certains ardents réformistes tels que Turgeon et Robidoux, ainsi qu'avec deux ardents défenseurs du régime de Mercier, A. Turgeon et G.-M. Dechêne.

Marchand était aussi prudent, même craintif, et se montra beaucoup plus un chef de centre droite qu'autre chose. Aux ouvriers de Montréal qui réclamaient avant l'élection des candidats qu'ils fussent vraiment représentatifs de leurs intérêts et non pas des notaires, médecins ou avocats, choisis par les gens de la rue St-Jacques et qui ne connaissaient rien à leurs problèmes, Marchand répondait "qu'il ne voulait pas de candidature ouvrière". ${ }^{5}$

Qui est F.-G. Marchand, ainsi que son ministre des Travaux publics, S.-N. Parent?

Parce qu'il alliait de nombreuses qualités personnelles et une vaste expérience parlementaire, F.-G. Marchand fut appelé à former le cabinet. Après la période tumultueuse que les Québécois avaient connue sous le régime Mercier, suivie d'une certaine instabilité économique durant le régime d'Edmund Flynn, le futur chef allait incarner l'assurance et la stabilité dans le gouvernement des affaires de la province. Homme de lettres, directeur de deux journaux de l'époque: le Temps et le Franco-Canadien, ainsi qu'auteur de quelques pièces dramatiques,

${ }^{5} L^{\prime} E v e ́ n e m e n t$, décembre 1897. 
le futur chef, par son érudition et son autorité sans rudesse, surplombait ses confrères de l'Assemblée. Sa carrière parlementaire avait été particulièrement brillante. Sous le régime Joly, il détenait le poste de secrétaire provincial. En 1879, on le retrouve ministre des terres et forêts et, à l'arrivée de Mercier, tout en se tenant un peu à l'écart, il occupa le poste de président de la Chambre. Dans l'opposition, de 1891 à son avènement au pouvoir en mai 1897 , il fut l'un des seuls à qui on pouvait faire appel pour redorer le blason terni du parti.

Simon-Napoléon Parent, dénommé le père de l'électricité au Québec et appelé, non sans causer de l'animosité chez certains libéraux, à remplacer son chef à sa mort subite le 25 septembre 1900 , fut le plus important lieutenant de Marchand. Sa présence au ministère était attribuable à plusieurs causes. Maire de la ville de Québec, président de la compagnie Dupont, administrateur au "Soleil" et à la "Quebec Railway Light and Power Company" ainsi qu'avocat-conseil à la banque Molson, Parent avait le champ libre pour manier le "patronage" à sa guise. Excellent administrateur, il pouvait se féliciter d'avoir augmenté, de 1897 à 1905 , le revenu du domaine public de $\$ 2,269,817$. sur le régime précédent, en imposant des taxes sur les rentes foncières, des droits de coupe de bois et des licences de pêche, de pouvoir d'eau et de grève. Sans aucun doute, il demeure l'un des grands initiateurs de l'essor industriel que connaîtra la province durant la fin d'un siècle et le début d'un autre.

\section{Le programme politique}

D'après le discours du trône du 23 novembre 1897, l'ère libérale s'annonça, sinon révolutionnaire, du moins très avantgardiste, précisément aux chapitres de l'instruction publique, de la colonisation et de l'exploitation des terres et des cours d'eau.

La substitution d'un ministère à la surintendance de l'Instruction publique, avec la gratuité scolaire au niveau des écoles primaires, préconisée par Emery Robidoux, allait soulever une guerre religieuse, ranimant d'anciennes querelles entre les gallicans et les ultramontains. Autour du projet de loi Marchand- 
Robidoux en faveur de la création d'un ministère, les quotidiens le Herald, la Patrie, le Pays, le Soleil et quelques autres, firent tous campagne en faveur d'une réforme. Ils exigèrent la suppression du Conseil, comme actuellement conçu, pour le remplacer par un ministère, avec droit pour le ministre de nommer ou de révoquer les inspecteurs. Le projet stipulait également moins de latin, d'histoire sainte et plus d'anglais et de science, ainsi que des cours obligatoires de dessin et d'agriculture dans les écoles rurales. Il fallait, selon les protagonistes du projet, arracher l'enseignement au clergé réactionnaire et retardataire, cesser de ne préparer que des séminaristes et avocats et favoriser la formation d'un plus grand nombre d'ingénieurs. On préconisait l'établissement d'écoles et de sociétés artistiques, littéraires et scientifiques.

Il n'en fallait pas moins pour piquer au vif les ultramontains qui ne tardèrent pas à se regrouper autour de NN. SS. Laflèche et Bruchési et de Thomas Chapais. Ils engagèrent le combat en accusant certaines gens d'anticléricalisme et de socialisme. Dans un retentissant plaidoyer en faveur du maintien du système actuel, Chapais dira:

et puis :

pour la laïcisation, ne voit-on pas que nous y marchons à grands pas ${ }^{6}$

Il y a des hommes dans la province qui veulent nous conduire au socialisme d'état en matière d'éducation. ${ }^{7}$

Mgr Bruchési se rendra à Rome afin de soumettre le litige au Pape. Le geste n'était pas nécessaire, car après avoir difficilement franchi les trois étapes en Chambre, le projet fut rejeté par le Conseil législatif.

\section{La frontière nord du Québec}

Le régime Marchand connut plus de succès en matière de colonisation qu'il n'en connaissait en éducation. Henry O'Sulli-

${ }^{6}$ Discours sur la loi de l'Instruction Publique, 1898, prononcé par l'honorable M. Chapais, 2. Bro. 15 pages.

7 Ibid., 3. 
van, arpenteur-inspecteur à l'emploi du gouvernement, après de nombreux voyages effectués dans la péninsule de la mer d'Hudson et des régions environnantes, concluait un rapport en disant que cette région regorgeait de charbon, de lignite, d'anthracite, de nombreux pouvoirs d'eau ainsi que de grandes richesses poissonnières, propices à l'établissement de centres industriels et de colonisation. Ne restait donc plus au régime Marchand que de s'en porter acquéreur.

Durant l'époque de Mercier, une délégation à Ottawa avait réclamé la rivière East Main comme frontière nord de la province, ce qui correspondait à la même latitude que la rivière Albany située à l'ouest de la baie James et limite officielle de la frontière nord de l'Ontario depuis 1878. Ottawa décidait, par ligne imaginaire, de tracer la frontière à 13 milles au sud de la rivière East Main. Mais aucun texte de loi n'allait confirmer cette nouvelle délimitation. Plus tard, par arrêté en conseil, le régime Flynn procédait, avec Ottawa, à la nouvelle répartition. Cela était encore insuffisant. Car seule la législature, en votant une loi, pouvait fixer les limites frontalières. Ce n'est qu'en 1898 , sous l'administration de Marchand, que le gouvernement se portera acquéreur du domaine de Rupert, embrassant quelques 67,499,930 acres de terre. Déjà on parlait d'un chemin de fer pour relier les régions du lac St-Jean et de la baie James, la distance entre les deux n'étant que de 380 milles. Et, au dire de plusieurs, cette route pourrait devenir la plus populaire pour atteindre les riches gisements d'or du Klondyke.

\section{Le Congrès de colonisation}

Ce congrès de novembre 1898 est révélateur en ce qu'il allait démontrer les profondes divergences d'opinions qui existaient autour du problème de l'exploitation de notre plus grande ressource à l'époque. L'école traditionnelle s'en prend ouvertement à la politique économique de Napoléon Parent. Il a vendu pour plus de $\$ 300,000$. en limites de terres aux magnats de l'industrie de la pulpe et du papier. Depuis vingt et même trente ans, disait-on, les influences politiques et les Canadiens anglais avaient eu raison du colon, en détenant les lots pour le seul 
avantage de la spéculation, refusant tout travail de défrichement pour n'attendre que le moment favorable de vendre à profit. La réforme des traditionalistes se voulait radicale, en réclamant instamment la création d'un conseil de colonisation entièrement soustrait aux influences politiques et nationales, tel que le conseil de l'Instruction publique l'était dans la tentative de réforme de l'éducation.

Par contre, le groupe de l'école moderne, avec Parent à sa tête, soutient que la vente des terres aux industriels est nécessaire pour le développement de l'industrie de la pâte et du papier et pour la mise en exploitation de nos incomparables pouvoirs hydrauliques. De la vente et de l'exploitation de ces ressources résulterait une valeur accrue pour chaque terre détenue par le colon. La présence de trois membres du cabinet à ce congrès démontre l'intérêt que le gouvernement portait à cette question vitale. Mais les efforts de Dechêne, Stevens et Robidoux iront vers la conciliation des groupes dissidents plutôt que vers l'acceptation d'une théorie au détriment de l'autre.

Peut-être ces hommes avaient-ils saisi la nécessité de concéder des terres à l'industrie et au commerce pour promouvoir une industrie secondaire dans la province ? Par contre, ils ne semblent pas avoir saisi l'état d'insécurité dans lequel une telle politique plongerait le colon et n'ont pas tenté l'impossible pour trouver des solutions adéquates à cette situation.

\section{La politique financière du gouvernement}

Marchand s'était présenté devant l'électorat en 1895 en alléguant que le régime d'Edmund Flynn, contrairement à ses promesses, endettait davantage la province. D'après le futur chef libéral, "la clique à Nantel" n'avait pu expliquer clairement où était allé plus de $\$ 3,727,027$. du revenu provincial, sur un total de $\$ 14,200,000$.:

Voici le ministre (Nantel) qui avec les siens et cinq ou six mignons a fourré la province pour $\$ 3,727,029$. en 4 ans. $^{8}$

${ }^{8}$ Le Soleil, 3 mai 1897, “Où sont les cliquards ?", 1, col. 2. 
Victor Gladu, député libéral de Yamaska, dévoila, avec chiffres à l'appui, que certains employés du ministère se payaient de fortes gratifications. Les amis et proches parents de Nantel auraient bénéficié de largesses pour des frais de voyages. Beemer, chef d'entreprise $d u$ chemin de fer du lac St-Jean, aurait reçu $\$ 1,486,506$. Ainsi s'expliquerait l'écart de $\$ 3,700,029$. dans les dépenses publiques.

Marchand préconisa un régime d'économie et prôna l'équilibre du budget. Citant les exemples des États de l'Ohio et de New-York qui, depuis 1873, réussissaient à réduire les impôts pendant que leurs populations augmentaient, il comptait réduire les dépenses. Et pour montrer les abus du gouvernement, il cite à nouveau l'exemple de nos voisins du sud, mais en empruntant cette fois-ci les chiffres de l'État fédéral qui, en 1887, ne dépensait en moyenne que $\$ 1.85$ par tête comparativement au Québec où la moyenne était de $\$ 3.75$. A son premier discours budgétaire pour l'année fiscale de 1897-98, Marchand se vantait d'avoir réduit le déficit annuel des dépenses publiques de $\$ 1,126,000$., le portant à moins de $\$ 239,000$. Et en 1900 , le trésorier Duffy se félicitait d'avoir presque balancé les revenus et les dépenses, chose qu'on n'avait pas vue depuis longtemps. Le surplus provenait de la location de lacs et de rivières et d'autres propriétés publiques. Cette politique de location du domaine public, exploitée à fond par Napoléon Parent, rapportait au trésor public la somme de $\$ 2,269,817$. D'autres montants provenaient de taxes directes, de taxes sur les corporations et des subsides fédéraux.

Pour avoir proposé des réformes en plusieurs domaines, afin de remédier aux injustices de l'époque, le régime de F.-G. Marchand a fait preuve de clairvoyance. Mais pour n'avoir pu mettre à exécution un projet d'éducation, si essentiel pour le relèvement de toute une société, faudrait-il en reporter l'accusation sur les membres trop conservateurs d'un Conseil législatif qui, comme conçu, n'avait et n'a toujours pas sa raison d'être, ou encore, pour les questions concernant l'éducation, sur la trop grande soumission au clergé de certains dirigeants de l'époque ?

BERNARD CHEVRIER 